\title{
METHODS OF DESIGNING GEAR'S MACHINING TOOLS WITH THE HYPERBOLOID CUTTING PART
}

\author{
VORONTSOV Boris ${ }^{1}$, BOSANSKY Miroslav ${ }^{2}$, KYRYCHENKO Iryna ${ }^{3}$, \\ KUZMENKO Nataliya ${ }^{3}$, STUPNYTSKYY Vadym ${ }^{4}$, KULESHKOV Yuriy ${ }^{5}$, \\ USTINENKO Alexander ${ }^{6}$ \\ ${ }^{I}$ National Technical University of Ukraine "Igor Sikorsky Kyiv Polytechnic Institute”, Ukraine \\ ${ }^{2}$ Slovak University of Technology in Bratislava, Faculty of Mechanical Engineering, Institute of Transport \\ Technology and Engineering Design, Nám. slobody 17,812 31 Bratislava, \\ Slovakia, e-mail: miroslav.bosansky@stuba.sk \\ ${ }^{3}$ Volodymyr Dahl East Ukrainian National University, Ukraine \\ ${ }^{4}$ Lviv Polytechnic National University, Ukraine \\ ${ }^{5}$ Central Ukrainian National Technical University, Ukraine \\ ${ }^{6}$ National Technical University “Kharkiv Polytechnical Institute”, Ukraine
}

\begin{abstract}
The technique of obtaining a hyperboloidal gear engagement with linear contact between a cylindrical involute wheel and a hyperboloid producing worm is considered. The resulting cutting tool greatly simplifies the manufacturing technology of hyperboloid worms and hyperboloid gears along with improved accuracy and roughness of the machined teeth and lower manufacturing costs.
\end{abstract}

KEYWORDS: gear, shaping pattern, toothed hyperboloid tool, profile, generating edge

\section{Introduction}

The efficiency of the machinery enterprises largely depends on the use of high-precision machining tools. Hobs are mainly used for cutting spur, bevel and worm gears. To reduce hobs wear, increasing their durability, productivity and accuracy a fundamentally new cutter cutting schemes necessary to create, that will ensure the most effective processing conditions. The new design of the gear machining tools with the hyperboloidal profile cutting part can be used to achieve this goal.

Procedures of designing hyperboloid cutters is the most important problem for the researchers, technologists and designers of the cutting tools. In this regard, the definition of the cylindrical toothing of a cylindrical generating wheel with a processed hyperboloid wheel and worm, as well as the definition of the machine meshing of a hyperboloid acting wheel with a cylindrical gear with linear contact is a problem that needs to be decided for the provision the trimming of the main surface with the generating surface.

This can be achieved by using existing cylindrical and rack tools for machining of the hyperboloid worms and hyperboloid wheels. Experimental and theoretical studies have shown that rolling tools in this case are performed on the cylindrical and hyperboloid workpiece without relief static angles, that is, the resulting tools without relieving. In unprofiled blade, the cutting face may be positioned at any rake angle depending on the hardness of the material being processed. Thus, the cutting force and power can be significantly reduced. The sharpening operation becomes secondary. The cutting edge, depending on the sharpening, can assume any spatial curve as long as it is on the generating surface. Thus, the accuracy of the tool will depend only on the accuracy of the main generating hyperboloid surface machining. 
This significantly reduces the cost of the tool and simplifies the technology for manufacturing hyperboloid worms and hyperboloid gears together with improving the accuracy and roughness of the teeth being machined.

\section{Review of literary sources}

Spatial machine engagement is mainly used to cylindrical wheels machining. However, tools working in spatial engagement are still imperfect, as they are designed either on cylinders (worm mills, rolling cutters) or planes (shaver-rack), although these surfaces should be single-sided hyperboloids $[1,2,3,4]$.

Currently, many studies for the purpose of reducing the cost of manufacturing spur gears are realized. As a result, several directions of enhancing durability and productivity of existing hobs $[5,6,7,8,9]$ which can be used in tools hyperboloid type are proposed.

The analysis of the stress-strain state of this tool can be performed by means of a Finite element method as it is described in [10]

The use of milling cutters with the rake and relief angles make a possibility to improve tool's durability. Relived cutter rake angles are $0^{\circ}-5^{\circ}$, the relief angles at the end cutting edge are $10^{\circ}-12^{\circ}$ and at the side cutting edge are $3^{\circ}-4^{\circ}$. The small rake angles do not provide high cutter's wear resistance. Increasing rake angles up to $10^{\circ}-15^{\circ}$ significantly improves stability, but leads to great disadvantages, such as: it reduces the possible number of regrinding, increasing error being cut wheel. In this case, an additional correction in the cutter tooth profile must be administered to reduce the error. But the errors of the corrected profile remain too large. This is the reason for limited use cutters with enhanced rake angles, resulting after regrinding to change of the generating surface and the worm cutter profile.

The analysis carried out of the main directions of increase of productivity in gear machining that although in recent years fundamental research has been carried out and many valuable results have been obtained, the problem of increase of productivity, accuracy and quality in toothing operations and reduction of consumption of expensive toothing cutters stay important.

The interest of engineers, researchers, designers and technologists to the gear cutting tools and gear systems with the crossing axes is not accidental. This is due to the desire to increase the load capacity of such gears. In some works, researchers try to propose an increase in the load capacity of gears through improvements based on theoretical studies, in others they propose improved results of profiling based on experimental works [11]. The analysis of such studies leads to the following conclusion: the main task set by most researchers is the task of compatible synthesis of gears and tool's support for processing the corresponding profile. Thus, in the spatial machine engagement, the main involute surfaces have a point-by-point touch character. When creating a cutting tool on this basis, the cutting edge must assume a defined spatial curve coinciding with the contact line of the contacting major surfaces. As a result, the rake angle is not the same as it should be based on the minimum cutting force condition. Such tools as shapers, roll cutters will be no cut the teeth gap on the crossing axis as they are fed along the axis of rotation of the workpiece. Therefore, it is a problem to find an acceptable cutting-edge structure by changing the side surfaces of the teeth (columns) of the hyperboloid main surfaces in order to obtain a linear touch character between the main surfaces (to increase load capacity).

Finishing of teeth after hardening in high-volume serial type of production, developed at Reishauer Co Ltd [12], is a high-performance operation. The disadvantage is that the columns of the hyperboloid grinding wheel are geometrically different when passing through the neck 
section. As a result, the surfaces to be treated will also be different along the length of the tooth. Therefore, the cutting tool must be made on half of the single-sided hyperboloid (at any rake angle, which reduces the cutting force).

\section{Goal research}

The purpose of the study is modelling and description of the machining engagement, consisting of generation spur or helical gear with involute profile or rack-type tool (cutter) and cutted hyperboloidal worm designed at the half hyperboloid type workpiece.

The following tasks are defined for achievement of a goal:

1. The creation of a hyperboloid gear mill based on the main hyperboloid tool worm,

2. Analytical profile description of the hyperboloid cutter in spatial machine engagement.

\section{The main material of the study. Hyperboloid surface equations that turn round the surface of the machining tool}

The hyperboloid worm can be machined as shown in Fig. 1. It follows from the figure that the rake cutting face of the machining cylindrical wheel is positioned parallel to the straight single-sided hyperboloid. Rotation of the hyperboloid worm and the cylindrical generating wheel or cutter feed is kinematically linked depending on number of teeth on wheels. In the case of a spur wheel, kinematic coupling is performed only by means of the dividing mechanism in the tool machine. In the case of a helical gearing, the kinematic coupling of the rotation of the mating surfaces is carried out by adjusting the index change gear train (depending on the number of teeth) and the differential change gear box (depending on the angle $\beta$ of inclination on the cylindrical wheel of the teeth). Cutting is performed by end section of cylindrical wheel.

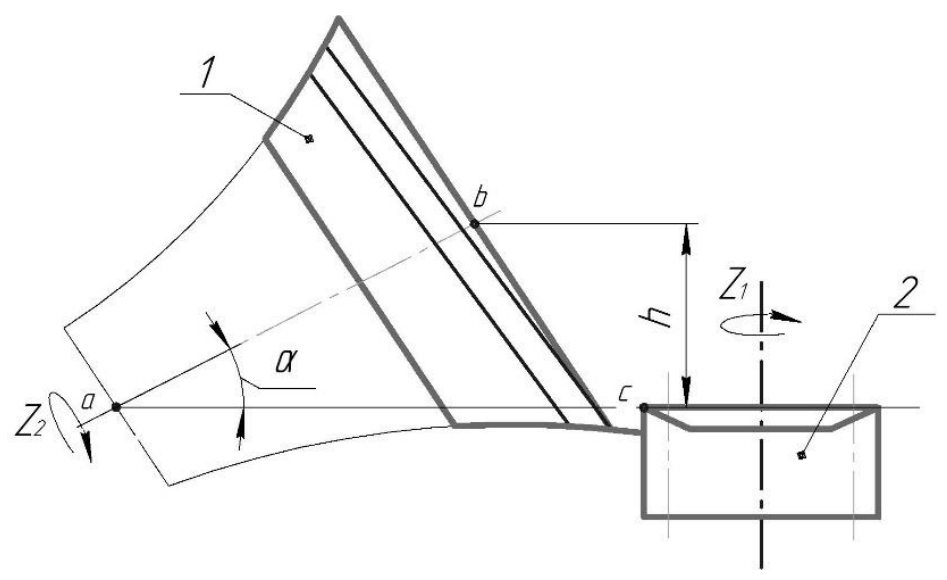

Fig. 1. Scheme of manufacturing of hyperboloid tool. 1 - workpiece; 2 - tool (circular shaper-type cutter)

The main cutting motion in this case is the relative sliding of the rake cutting edges of the tool cylindrical wheel or the normal tooth section of the helical wheel against the cut turns on the hyperboloid workpiece.

In cutting, when the feed and rolling parameters $\mu(S)$ and $\phi_{1}$ are not time related, the equation is as follows: 
$\left.\begin{array}{l}x_{2}=\left(f_{1}-r_{1}\right)\left(\cos \phi_{1} \cos \phi_{2}+\cos \gamma \sin \phi_{1} \sin \phi_{2}\right)+f_{2}\left(\cos \gamma \cos \phi_{1} \sin \phi_{2}-\right. \\ \left.-\sin \phi_{1} \cos \phi_{2}\right)-\mu \sin \gamma \sin \phi_{2}+a_{w} \cos \phi_{2} ; \\ y_{2}=\left(f_{1}-r_{1}\right)\left(\cos \gamma \sin \phi_{1} \cos \phi_{2}-\sin \phi_{2} \cos \phi_{1}\right)+f_{2}\left(\sin \phi_{1} \sin \phi_{2}+\right. \\ \left.+\cos \gamma \cos \phi_{1} \cos \phi_{2}\right)-\mu \sin \gamma \cos \phi_{2}-a_{w} \sin \phi_{2} ; \\ \left.z_{2}=\left(f_{1}-r_{1}\right) \sin \gamma \sin \phi_{1}\right)+f_{2} \sin \gamma \cos \phi_{1}+\mu \cos \gamma .\end{array}\right\}$

These equations describe the surfaces of hyperboloid worm turns formed by contact lines (cutting profiles).

Thus, when the feed and rotations of the tool and workpiece are not time bound together, the equations (1) define the surfaces of the hyperboloid worm turns (from which the hyperboloid worm cutter formed by the cutting edges of the cylindrical wheel will be profiled in the following). In general, these surfaces will differ the less from the mutually enveloping surfaces with the surfaces of the cylindrical gear wheel, then less the feed of the cylindrical wheel determined by the parameter $\mu(S)$.

To match said surfaces, it is necessary and sufficiently to equal zero of the mixed product, which is the engagement equation of the form:

$$
\left[\vec{t}_{1}, \vec{V}^{(1 \mu)}, \vec{V}^{(12)}\right]=0
$$

where $\vec{t}_{1}$ is the vector tangent to the tooth profile of the cylindrical wheel;

$\vec{V}^{(1 \mu)}$ is the speed of tooth profile movement along straight generatrix of single-sided hyperboloid;

$\vec{V}^{(12)}$ is the speed of the cylindrical wheel tooth profile movement relative to hyperboloid worm turn if value of parameter $\mu$ is fixed.

The tangent vector $t_{1}$ to the cylindrical wheel profile in the end section can be written as follows:

$$
\vec{r}_{1}^{\lambda}=\vec{t}_{1}=f_{1}^{\prime}(\lambda) \vec{i}+f_{2}^{\prime}(\lambda) \vec{j}
$$

where $f_{1}{ }^{\prime} \lambda, f_{2}{ }^{\prime} \lambda$ are the derived functions by tooth profile angle of generating cylindrical wheel.

Projections of relative velocity of the point located on the profile of the cylindrical wheel tooth at a fixed value and variable angle in the movable coordinate system can be written by the system of equations (4).

$$
\left.\begin{array}{l}
\vartheta_{x 1}{ }^{(12)}=\left(f_{1} \sin \phi_{1}-r_{1} \sin \phi_{1}-f_{2} \cos \phi_{1}\right)\left(1-u_{21} \cos \gamma\right)- \\
-\mu u_{21} \sin \gamma \cos \phi_{1}-a_{w} u_{21} \cos \gamma \sin \phi_{1}, \\
\vartheta_{y 1}{ }^{(12)}=\left(f_{1} \cos \phi_{1}-r_{1} \cos \phi_{1}+f_{2} \sin \phi_{1}\right)\left(1-u_{21} \cos \gamma\right)+ \\
+\mu u_{21} \sin \gamma \sin \phi_{1}-a_{w} u_{21} \cos \gamma \cos \phi_{1}, \\
\vartheta_{z 1}{ }^{(12)}=u_{21} \sin \gamma\left(f_{1}-r_{1}\right)+a_{w} .
\end{array}\right\}
$$

Vector of relative speed motion of the tooth profile point at the cylindrical wheel (cutting edge) at a fixed parameter $\phi_{1}$ can be described as equations (5). 


$$
\vec{V}^{(1 \mu)}=d \mu / d t \vec{k}
$$

Substituting the vector values $\vec{t}_{1}, \vec{V}^{(12)}, \vec{V}^{(1 \mu)}$ from the equations (3), (4) into (2) gives the mixed vector product as equations (6).

$$
\left[\vec{t}_{1}, \vec{V}^{(1 \mu)}, \vec{V}^{(12)}\right]=\vec{t}_{1}\left[\vec{V}^{(1 \mu)} \times \vec{V}^{(12)}\right]
$$

where

$$
\begin{gathered}
{\left[\vec{V}^{(1 \mu)} \times \vec{V}^{(12)}\right]=i V_{y 1}{ }^{(1 \mu)} V_{z 1}{ }^{(12)}+j V_{z 1}{ }^{(1 \mu)} V_{x 1}{ }^{(12)}+} \\
+k V_{x 1}{ }^{(1 \mu)} V_{y 1}{ }^{(12)}-k V_{y 1}{ }^{(1 \mu)} V_{x 1}{ }^{(12)}-i V_{z 1}{ }^{(1 \mu)} V_{y 1}{ }^{(12)}-j V_{x 1}{ }^{(1 \mu)} V_{z 1}{ }^{(12)} .
\end{gathered}
$$

As a result of the transformations of formula (6) taking into account equations (3), (5), we obtain:

$$
f_{1}^{\prime} \lambda V_{z 1}^{(1 \mu)} V_{y 1}^{(12)}+f_{2}^{\prime} \lambda V_{z 1}^{(1 \mu)} V_{x 1}^{(12)}=0 .
$$

From formulas (4), (5), (7) we receive the equations of correlation (2) between parameters $\mu, \lambda, \phi_{1}$ as follows:

$$
\begin{aligned}
& f_{1}^{\prime}(\lambda) \partial \mu / \partial t\left[\left(f_{1}(\lambda)-r_{1}\right)\left(1-u_{21} \cos \gamma\right)+\mu u_{21} \sin \gamma \sin \phi_{1}-a_{w} u_{21} \cos \gamma \cos \phi_{1}\right]+ \\
& +f_{2}^{\prime}(\lambda) \partial \mu / \partial t\left[-f_{2}(\lambda)\left(1-u_{21} \cos \gamma\right)-\mu u_{21} \sin \gamma \cos \phi_{1}-a_{w} u_{21} \cos \gamma \sin \phi_{1}\right]=0 .
\end{aligned}
$$

By reducing by a common multiplier $d \mu / d t$ the left part of this equation and by making the transformation, we get the engagement equations between the parameters $\lambda, \mu, \phi_{1}$ as follows:

$$
\begin{gathered}
F\left(\lambda, \mu, \phi_{1}\right)=-f_{1} \partial \mu / \partial t\left[\left(f_{1}-r_{1}\right)\left(1-u_{21} \cos \gamma\right)+\mu u_{21} \sin \gamma \sin \phi_{1}-\right. \\
\left.-a_{w} u_{21} \cos \gamma \cos \phi_{1}\right]-f_{2}^{\prime} \partial \mu / \partial t\left[f_{2}\left(1-u_{21} \cos \gamma\right)-\right. \\
\left.-\mu u_{21} \sin \gamma \cos \phi_{1}-a_{w} u_{21} \cos \gamma \sin \phi_{1}\right]=0 .
\end{gathered}
$$

The machine engagement equation fully matches equations (8). This means that the surface of the hyperboloid coil described by this system of equations is similar to that of the hyperboloid coil obtained as a result of the forming motion of the tool's cutting edge.

Note here, that surface of hyperboloid turn has linear character of touching with cylindrical wheel tooth. Cutting edge on side surface of cylindrical wheel tooth makes only point touch.

It follows from the above, that if for the cutting edge been located on the tooth of the cylindrical wheel and for the hyperboloid workpiece will be given a rolling motion, the cutting edge will leave only a outer track as a contact line on the machined workpiece surface and will not reproduce the cutting motion.

In order to form a machine engagement, it is necessary, in addition to the rolling 
movements of the article and the cutting edge, to give an additional swinging motion of the tool along the straight generatrix of the hyperboloid.

Equation (8) defines one of the variables. When calculated it relative to the parameter $\mu$, we get the following [9]:

$$
\begin{gathered}
\mu=-a_{w} u_{21} \cos \gamma\left[f_{2}^{\prime} \sin \phi_{1}-f_{1}^{\prime} \cos \phi_{1}\right]+\left(1-u_{21} \cos \gamma\right)\left[f_{2} f_{2}^{\prime}+\right. \\
\left.+f^{\prime}+f_{1}(\lambda)\right] /\left[u_{21} \sin \gamma f_{2}^{\prime}\left(\cos \phi_{1}+f_{1}^{\prime} \sin \phi_{1}\right)\right] .
\end{gathered}
$$

The engagement surface equations are obtained if writing the coordinates of the cylindrical wheel tooth surface in a fixed coordinate system and using the parameter values $\mu$ from equation (9). Applying the transition formulas from a mobile coordinate system $S_{1}\left(x_{1} y_{1} z_{1}\right)$ to a fixed coordinate system $S(x y z)$, we get the engagement surface equations as follows:

a) for the spur gear:

$$
\begin{gathered}
x=f_{1} \cos \phi_{1}-r_{1} \cos \phi_{1}-f_{2} \sin \phi_{1} \\
y=f_{1} \sin \phi_{1}-r_{1} \sin \phi_{1}+f_{2} \cos \phi_{1} \\
z=-a_{w} u_{21} \cos \gamma\left(f_{2}^{\prime} \sin \phi_{1}-f_{1}^{\prime} \cos \phi_{1}\right)+\left(1-u_{21} \cos \gamma\right)\left(f_{2} f_{2}^{\prime}+\right. \\
\left.\left.+f_{1}^{\prime} f_{1}-r_{1} f_{1}^{\prime}\right)\right] /\left[u_{21} \sin \gamma\left(f_{2}^{\prime} \cos \phi_{1}+f_{1}^{\prime} \sin \phi_{1}\right)\right] .
\end{gathered}
$$

b) for the helical gear:

$$
\begin{gathered}
x=f_{1} \cos \left(\mu+\phi_{1}\right)-f_{2} \sin \left(\mu+\phi_{1}\right) ; \\
y=f_{1} \sin \left(\mu+\phi_{1}\right)+f_{2} \cos \left(\mu+\phi_{1}\right) ; \\
F\left(\theta, \mu, \phi_{1}\right)=P^{2} \mu\left[f_{1}^{\prime} \sin \left(\mu+\phi_{1}\right)+f_{2}^{\prime} \cos \left(\mu+\phi_{1}\right)\right]+P a_{w}\left[f_{2}^{\prime} \sin \left(\mu+\phi_{1}\right)\right. \\
\left.-f_{1}^{\prime} \cos \left(\mu+\phi_{1}\right)\right] \operatorname{ctg} \gamma-\left[f_{1} \cos \left(\mu+\phi_{1}\right)-f_{2} \sin \left(\mu+\phi_{1}\right)+a_{w}-\right. \\
\left.\left.-P\left(1-u_{21} \cos \gamma\right)\right] / u_{21} \sin \gamma\right]\left(f_{1}^{\prime} f_{1}+f_{2} f_{2}^{\prime}\right) .
\end{gathered}
$$

Let's define the line of contact engagement with hyperboloid cylindrical worm wheel in the fixed coordinate system at a fixed value $\varphi_{1}$ (in the equations (10) and (11)).

Let the mating surface belonging to the cylindrical wheel $z_{1}$ be the surface of the article. If another hyperboloid worm $z_{2}$ whose side surface of the turns is conjugated with the surface of the teeth of the cylindrical wheel $z_{1}$ is converted into a tool with cutting edges, then, if there is linear contact (equations 10 and 11) of the wheel teeth surfaces $z_{1}$ and $z_{2}$ or vice versa $z_{2}$ with $z_{1}$, as the cutting edge of the tool on the wheel teeth surface $z_{1}$ or $z_{2}$ whichever is an workpiece (cylindrical or hyperboloid worm) can take any line, as long as it has points throughout the active side of the tooth involved in the engagement.

If you take any line on the surface of the hyperboloid turn $z_{2}$ as the cutting edge of the tool, located on the active part of the tooth, if there are movements of tool and article rolling, the cutting edge of the tool during rolling will have contact with the surface of the article due to the fact that the cutting edge lies on the surface, any point of which, due to linear contact, has contact at a certain moment of rolling with the formed surface of the workpiece [2].

Consequently, this cutting edge will also leave a line of contact trace on the surface of the workpiece. Thus, if there is additional movement, in addition to the rolling movements, we will get a grid of contact lines that will be located on the surface of the workpiece, in other words, we will get the machined gear profile. 
If there is a kinematic pair with linear contact of mating surfaces of teeth, only lines be located on the surface of turns of one of elements of the kinematic pair can be taken as cutting edges.

Suppose you have a line as the cutting edge of the tool that does not be located on the surface of one of the mated surfaces with a linear contact. Then, it is apparent that the cutting edge cannot leave a line of contact with the other mating surface in motion, since such a cutting edge will be located outside the surfaces at any time of contact of the mating surfaces along the line, will not touch them at any point, and therefore cannot form a predetermined mating surface by the rolling method.

\section{CONCLUSION}

1. Received hyperboloidal machine gearing with linear contact between a cylindrical involute wheel and a hyperboloid producing worm.

2. Received a hyperboloid worm on a rectilinear generatrix of which the cutting edges are located.

3. It is proved that the main hyperboloid surfaces are obtained with the help of cylindrical involute wheels in spatial machine engagement, when the generatrix of the cylindrical wheel is perpendicular to the straight one-sheeted hyperboloid. In this case, the contact nature of the mating surfaces is linear. This means that the front cutting face on the hyperboloid milling cutter can be inclined at any angle depending on the material being processed and its hardness. In this case, the geometric distortion of the cutting edges does not affect the accuracy of the machined teeth. This is due to the lack of technological operations of backing of the tool in question.

\section{REFERENCES}

[1] Vorontsov, B. S. "Computer-integrated system for ensuring the formation of gears". The dissertation of a doctor of technical sciences: 05.03.01 - machining processes, machines and tools. National Technical University of Ukraine "Igor Sikorsky Kyiv Polytechnic Institute”, Kyiv, Ukraine, 2019. - 397 p.

[2] Kirichenko, I. A. "Creation of hyperboloid gears with linear contact of teeth on the basis of special cutting tools". The dissertation of a doctor of technical sciences: 05.02.02 machine science. Volodymyr Dahl East Ukrainian National University, Lugansk, Ukraine, 2004. $-274 \mathrm{p}$.

[3] Gavrilenko, V. A. "Gears In mechanical engineering", Mashgiz, Moscow, USSR, 1968, $280 \mathrm{p}$.

[4] Kashura, A. L., Vorontsov, B. S., Kirichenko, I. A. "The shaping of quasi-globoid worm mills", Visnik "KhPI", No. 28, pp.76 - 82, 2011.

[5] Bodzás, S. "Analysis of the Effect of the Addendum Modification Coefficient for Contact Surfaces of Spur Gear", Strojnícky časopis - Journal of Mechanical Engineering 69 (1), pp. 5 - 16, 2019. DOI: 10.2478/scjme-2019-0001

[6] Kuzmenko, N. N., Kirichenko, I. A. "The manufacture of gears and milling cutters on single-cavity hyperboloids", Visnik SNU im. V. Dalya. No 1 (242), pp.73 - 77, 2018.

[7] Vorontsov, B. S., Kirichenko, I. A., Vitrenko, O. S. "The study of the process of forming hyperboloid gears using a tool gear", Instrument reliability and of technological systems, No. 30, pp. 188 - 194, 2012. 
[8] Vorontsov, B. S., Bocharova I. A., Vitrenko V. A., Kirichenko I. A. "Controlled synthesis of gears with intersecting axes", News of SNU im. V. Dalya, №7 (89), pp. 34 - 36, 2005.

[9] Gribanov, V. M. "Gear hyperboloid gears", News of SNU im. V. Dalya, No.2 (36), pp.123 $-133,2001$.

[10] Trebuňa, F., Bocko, J., Pástor, M., Lengvarský, P., Prada, E. "The Finite Element Analysis of High Precision Positioning System", Strojnícky časopis - Journal of Mechanical Engineering 68 (4), pp. 41 - 48, 2018. DOI: 10.2478/scjme-2018-0045

[11] Kirichenko, I. A., Vitrenko, V. A., Doan Duc Vinh. "Milling cutters for milling involute teeth of cylindrical wheels, based on third-class shaping schemes", Bulletin of the Kharkov State Polytechnical University, No. 85, pp.57 - 65, 1999.

[12] Prospectus of the REYSHAUER company. Economic final processing of teeth after hardening in large-scale production, 91 p. 1991. 